\title{
Altered functional connectivity of the amygdaloid input nuclei in adolescents and young adults with autism spectrum disorder: a resting state $\mathrm{fMRI}$ study
}

\author{
Annika Rausch ${ }^{1,2^{*}}$, Wei Zhang ${ }^{2}$, Koen V. Haak ${ }^{2}$, Maarten Mennes ${ }^{2}$, Erno J. Hermans ${ }^{1,2}$, Erik van Oort ${ }^{2,3}$,
} Guido van Wingen ${ }^{2,4}$, Christian F. Beckmann ${ }^{1,2,5}$, Jan K. Buitelaar ${ }^{1,2,6}$ and Wouter B. Groen ${ }^{2,6}$

\begin{abstract}
Background: Amygdala dysfunction is hypothesized to underlie the social deficits observed in autism spectrum disorders (ASD). However, the neurobiological basis of this hypothesis is underspecified because it is unknown whether ASD relates to abnormalities of the amygdaloid input or output nuclei. Here, we investigated the functional connectivity of the amygdaloid social-perceptual input nuclei and emotion-regulation output nuclei in ASD versus controls.

Methods: We collected resting state functional magnetic resonance imaging (fMRI) data, tailored to provide optimal sensitivity in the amygdala as well as the neocortex, in 20 adolescents and young adults with ASD and 25 matched controls. We performed a regular correlation analysis between the entire amygdala (EA) and the whole brain and used a partial correlation analysis to investigate whole-brain functional connectivity uniquely related to each of the amygdaloid subregions.

Results: Between-group comparison of regular EA correlations showed significantly reduced connectivity in visuospatial and superior parietal areas in ASD compared to controls. Partial correlation analysis revealed that this effect was driven by the left superficial and right laterobasal input subregions, but not the centromedial output nuclei.
\end{abstract}

Conclusions: These results indicate reduced connectivity of specifically the amygdaloid sensory input channels in ASD, suggesting that abnormal amygdalo-cortical connectivity can be traced down to the socio-perceptual pathways.

Keywords: Amygdala, Autism spectrum disorder, Centromedial, Connectivity, Input-output, Laterobasal, Nuclei, Social perception, Superficial

\section{Background}

Autism spectrum disorders (ASD) are a group of neurodevelopmental disorders characterized by severe impairments of reciprocal social interaction and verbal and nonverbal communication and by repetitive and stereotyped behaviors [1, 2]. Structural and functional neuroimaging studies have linked a number of brain

\footnotetext{
* Correspondence: a.rausch@psych.ru.nl

${ }^{1}$ Department of Cognitive Neuroscience, Radboud University Medical Center Nijmegen, P.O. Box 91016500 HB Nijmegen, The Netherlands

${ }^{2}$ Donders Institute for Brain, Cognition and Behaviour, Centre for Cognitive Neuroimaging, Radboud University, Nijmegen, The Netherlands

Full list of author information is available at the end of the article
}

structures to ASD symptoms [3-6], one of which is the amygdala. The amygdala theory of autism describes this structure as potential key component in the pathogenesis of ASD [7, 8], since it is involved inwfi various aspects of the social brain, such as social cognition, emotion recognition, sociocommunicative perception, and the regulation of emotional responses [9]. In line with this, individuals with ASD tend to show abnormal volume enlargements of the amygdala $[10,11]$ and have overactive amygdalae in response to mildly aversive stimuli [12] and faces [13], while symptom severity in ASD has been found to correlate with amygdala size $[10,14,15]$. Although 
amygdala impairments likely relate to pathophysiological socio-emotional processes, its subregionspecific amygdalo-cortical abnormalities have not been stratified in ASD. The aim of this study is to advance our understanding of the pathway-specific amygdala involvement and discern sensory input and response output channels separately.

With the last decade's paradigm shift in neuroimaging from activity assessment within brain structures to connectivity within neural networks, increasing evidence supports the notion of atypical large-scale neural connectivity in ASD. Some authors hypothesized that the brain in ASD is characterized by long distance underconnectivity and local overconnectivity [16]. Indeed, a number of studies reported large-scale underconnectivity with decreased structural, functional, and interhemispheric connectivity $[4,17,18]$, and a few functional magnetic resonance imaging (fMRI) studies found local overconnectivity patterns [19-23]. To date, large-scale functional connectivity abnormalities in the autistic brain have been described most conclusively with respect to hyper- or hyposensitivity to perceptual stimuli [24], which may in part occur due to a lack of sensory integration in ASD [4]. Abnormal sensory processing has primarily been found in the auditory [25-27] and visual system [28-30]. Because social functioning requires the selection and integration of many socio-perceptual stimuli simultaneously, perceptual processing abnormalities may in part account for some of the social difficulties in ASD.

Previous fMRI studies on the role of the amygdala in ASD have generally treated it as a single structure, while in fact it is comprised of at least 13 functionally and structurally distinct nuclei, in which three major input and output units can be discerned [31-33]: the centromedial (CM), laterobasal (LB), and superficial (SF) nuclei. Prior work in animals identified the centromedial part as an output area, which regulates cardiovascular control via projections to the brainstem, cerebellum, and hypothalamus [34]. More specifically, the $\mathrm{CM}$ subregion generates ascending projections via the forebrain throughout the cortex and descending projections via the hypothalamus to the brainstem [35]. Via these complex pathways, the CM subregion is thought to modulate autonomic, somatic, and endocrine responses to facilitate appropriate behavioral outcome [36]. A recent study in humans mapped projections from the cortical social "aversion network", a network that is situated around the anterior cingulated cortex, onto a functionally defined amygdaloid subdivision that corresponds to the CM subcompartment [37]. This subdivision may therefore be associated with emotion regulation and response preparation in humans as well [38]. The CM subcompartment receives and integrates most of its projections from the LB nuclei, which maintains broad axonal connections to sensory areas. The LB has been linked to multisensory input and emotional learning [9, 38, 39], especially emotional memory [38]. The SF subregion primarily maintains axonal projections to olfactory cortex [38, 40,41], and it was found to be more sensitive to emotional face recognition than LB and CM [42], as well as to maintain the most behavioral correlates of the three amygdaloid subregions [38]. The LB and SF comprise structurally and functionally clearly differentiable properties: while the LB mainly receives multisensory environmental input, the SF is thought to receive socially relevant information [43]. Yet, they are often mentioned together as the olfactory/multimodal pallial section of the amygdala as both structures generally process incoming stimuli [32], so as to facilitate social-perceptual processing [44].

Our study specifically aimed to investigate global networks in ASD. Since we consider amygdalo-cortical connectivity long-range connectivity, our hypotheses were aimed at underconnectivity. Given the prominent role of the amygdala in the social brain and previous findings of long-range underconnectivity in sensory areas in ASD, we hypothesized to find reduced functional amygdalo-cortical connectivity in ASD, especially among the projections from sensory cortex to the amygdala. We applied dual-echo imaging and a stringent correction for heart rate and respiratory signals to ensure optimal sensitivity in both the amygdala and neocortical structures.

\section{Methods}

\section{Participants}

Twenty-one adolescents with autistic disorder and 25 typically developing controls were enrolled in the study. We only included participants with an intelligence quotient (full-scale IQ) of 80 or higher and excluded those with ASD who had co-morbid psychiatric or neurological conditions including but not limited to attention deficit/hyperactivity disorder (ADHD), depressive disorder, schizophrenia, epilepsy, or history of traumatic brain injury. We ruled out the presence of psychiatric co-morbidity in controls and verified that all participants scored within the normal range using the school-age version of Child Behavior Check List (CBCL/ 6-18) and Adult Behavior Check List (ABCL/18-59). Controls were matched at the group level on age, sex, and handedness and verbal, performance, and full-scale IQ scores (Table 1). Participants with ASD were recruited through Karakter, Child and Adolescent Psychiatry University Center, Nijmegen. Diagnoses of autistic disorder were based on a series of clinical assessments including a detailed developmental history, clinical observation, 
Table 1 Subject demographics

\begin{tabular}{|c|c|c|c|c|c|}
\hline \multirow[b]{2}{*}{ Males } & \multicolumn{2}{|l|}{ ASD } & \multicolumn{2}{|c|}{ Control } & \multirow[b]{4}{*}{$p$ value } \\
\hline & \multicolumn{2}{|c|}{$N=19(95 \%)$} & \multicolumn{2}{|c|}{$N=22(88 \%)$} & \\
\hline \multirow[t]{2}{*}{ Females } & \multicolumn{2}{|c|}{$N=1(5 \%)$} & \multicolumn{2}{|c|}{$N=3(12 \%)$} & \\
\hline & Mean & SD & Mean & SD & \\
\hline Total IQ & 102.30 & 13.57 & 103.72 & 9.78 & 0.69 \\
\hline Verbal IQ & 101.00 & 13.37 & 104.60 & 11.29 & 0.35 \\
\hline Performal IQ & 105.88 & 15.81 & 103.00 & 15.39 & 0.56 \\
\hline Age & 16.23 & 3.18 & 16.11 & 2.79 & 0.90 \\
\hline \multicolumn{6}{|l|}{ Autism Questionnaire (AQ) } \\
\hline Participants & 21.83 & 6.13 & 11.88 & 3.91 & $<0.001^{*}$ \\
\hline Parents about participant & 30.34 & 7.57 & 11.74 & 5.69 & $<0.001^{*}$ \\
\hline \multicolumn{6}{|c|}{ Autism Diagnostic Interview (ADI-R) } \\
\hline ADI-R A (10) & 18.25 & 6.50 & & & \\
\hline ADI-R B (8) & 15.70 & 5.54 & & & \\
\hline ADI-R C (3) & 4.05 & 2.31 & & & \\
\hline ADI-R D (1) & 2.65 & 1.35 & & & \\
\hline
\end{tabular}

ADI-R thresholds are shown in parentheses. Pearson chi-squared for group by gender was nonsignificant (value $=0.672, d f=1$, two-sided asymptotic $p=0.412)$ $p$ values indicate results for the independent $t$ test statistic. $A D I-R A$ social interaction, $B$ communication and language, $C$ restricted and repetitive behavior, $D$ age of onset criterium

*Statistically significant

medical work-up, and cognitive testing in a multidisciplinary team including a child psychiatrist and clinical psychologist. Diagnoses of autistic disorder were confirmed with the Autism Diagnostic InterviewRevised (ADI-R) [45], assessed by a trained clinician who met research standards. All participants with ASD met DSM-4 criteria for autistic disorder [1]. Participants under the age of 18 completed the Wechsler Intelligence Scale for Children III (WISC-III) [46], while participants above the age of 18 completed the Wechsler Adult Intelligence Scale III (WAIS-III) [47]. All participants also completed the short version of Edinburgh Handedness Inventory [48]. The two groups did not differ in handedness $(p=0.17)$. In addition, all participants and their parents completed the autism spectrum quotient (AQ) about themselves or their child, respectively. The AQ is a validated measure of autism spectrum characteristics found within both the typical population and individuals with a diagnosis of ASD and thus provides a reliable measurement tool for the comparison of autistic traits between our ASD and control sample $[49,50]$. None of the participants used medication.

The study (including the informed consent procedure and all information brochures) was approved by both the regional ethics committee (Commissie Mensgebonden Onderzoek Arnhem Nijmegen) and Karakter's review board. All participants provided verbal and written informed consent. For underage participants, parents/ guardians also provided verbal and written informed consent. The signed consent forms are kept at Karakter, Child and Adolescent Psychiatry Centre Nijmegen, The Netherlands. To ensure an adequate consent procedure, (1) potential participants were provided with simple language brochures (parents/guardians were provided with regular brochures), (2) the study was not advertised with financial or other incentives other than travel reimbursement (after the scanning procedure, all participants did receive 20 euros for participating irrespective of completion), (3) participants were reminded at each phase of the study that they were free to withdraw from participating, (4) only participants with a total IQ of 80 or higher could participate, and (5) all participants practiced once with the scanning procedure in a replicate (dummy) scanner so that they could experience the scanning procedure and make an informed decision on whether or not to participate.

\section{Image data acquisition}

For each participant, we acquired magnetic resonance imaging (MRI) data at the Donders Institute for Brain, Cognition and Behaviour, Centre for Cognitive Neuroimaging in Nijmegen, The Netherlands, using a 3 Tesla Magnetom Trio (Siemens, Erlangen, Germany) with a 32-channel head coil. The entire scanning session lasted approximately $45 \mathrm{~min}$. For each participant, we collected a T1weighted whole-brain scan (magnetization-prepared rapid acquisition with gradient echo (MPRAGE), inversion time $(\mathrm{TI})=1100 \mathrm{~ms}$, repetition time $(\mathrm{TR})=2300 \mathrm{~ms}$, echo time $(\mathrm{TE})=3.03 \mathrm{~ms}$, flip angle $=8^{\circ}$, field of view $(\mathrm{FOV})=256 \times$ 
$256 \times 192 \mathrm{~mm}^{3}$, voxel size $=1 \times 1 \times 1 \mathrm{~mm}^{3}$ ) and a resting state scan using $\mathrm{T} 2 \%$-weighted dual-echo planar imaging (EPI, TR $=2510 \mathrm{~ms}, \mathrm{TE} 1=16 \mathrm{~ms}, \mathrm{TE} 2=36 \mathrm{~ms}$, flip angle $=83^{\circ}, \mathrm{FOV}=212 \times 212 \times 119 \mathrm{~mm}^{3}$, voxel size $=2 \times 2 \times$ $2.5 \mathrm{~mm}^{3}$, number of volumes $=400$, imaging bandwidth $=$ $1814 \mathrm{~Hz} / \mathrm{px}$, grappa acceleration factor $=4$ ). Note that the usage of dual-echo imaging provides optimal sensitivity for blood-oxygen-level-dependent (BOLD) imaging in both subcortical structures such as the amygdala and the neocortex [51]. For the 16-min resting state scan, participants were instructed to lie still within the scanner with their eyes open, while staying awake and focusing on a small white cross presented at the centre of a projection screen. The first five volumes (12.55 s) were discarded to reduce magnetization equilibration effects. Gradient echo field mapping data were also acquired with identical geometry to the EPI data for EPI off-resonance distortion correction $(\mathrm{TR}=1020 \mathrm{~ms}, \mathrm{TE} 1=10 \mathrm{~ms}, \mathrm{TE} 2=12.46 \mathrm{~ms}$, flip angle $=90^{\circ}, \mathrm{FOV}=224 \times 224 \times 191 \mathrm{~mm}^{3}$, voxel size $=$ $3.5 \times 3.5 \times 2 \mathrm{~mm}^{3}$ ). All participants were able to familiarize themselves with scanner setup and scanning procedure through rehearsal in a replicate (dummy) scanner before actual image acquisition. The scanning session further also included a diffusion tensor imaging (DTI) scan (not reported here).

We recorded participants' heartbeats using the scanner's built-in photoplethysmograph, placed on the right index finger. Respiration was measured with a pneumatic belt positioned at the level of the abdomen. In order to reduce the potential bias that the heartbeat and respiration have in resting state BOLD correlation studies [52, 53], we used cardiac and respiratory phase regressors, as well as other nuisance regressors in the fMRI time series analysis.

\section{Preprocessing}

All image preprocessing and analyses were performed using FSL (FMRIB Software Library, http://fsl.fmrib.ox. ac.uk/fsl/fslwiki/) [54]. The following pre-statistical processes were applied to the fMRI data: nonbrain removal using Brain Extraction Tool (BET); rigid-body motion correction using MCFLIRT; high-pass temporal filtering (Gaussian-weighted least-squares fitting with frequency cutoff point $=100 \mathrm{~s}$ ); correction of off-resonance geometric distortions in the EPI data using PRELUDE and FUGUE, using $B_{0}$ field maps derived from the dual-echo gradient echo dataset; artifact removal based on probabilistic ICA (Independent Component Analysis) using MELODIC; spatial normalization to Montreal Neurological Institute (MNI152) $2 \mathrm{~mm}$ isotropic atlas space using boundary-based registration (BBR); and FNIRT and Gaussian filtering (full width at half maximum $(\mathrm{FWHM})=6 \mathrm{~mm}$; see the the "Statistical analysis" section). The dual-echo images ( $\mathrm{TE}=16$ and $\mathrm{TE}=36$ ) were combined by averaging both echo times. We excluded one participant with ASD due to excessive head movement in terms of frame-wise displacement (max. framewise displacement $(\mathrm{FD})=8.7 \mathrm{~mm}, M_{\mathrm{fd}}=0.89 \mathrm{~mm}$ ), resulting in 20 datasets from the ASD group and 25 datasets from the control group for further analysis (see Additional file 1 for relative frame-wise displacement). To rule out the possibility that differences in movement between the ASD and control group could contribute to the results, we calculated the mean value of frame-wise movement (i.e., the movement of one TR relative to previous TR) for each participant and compared it between the two groups. No group difference was found $\left(M_{\text {asd }}=0.10, \quad \mathrm{SD}_{\text {asd }}=0.10 ; \quad M_{\text {ctrl }}=0.07\right.$, $\left.\mathrm{SD}_{\text {ctrl }}=0.42 ; t(25)=1.73, p=0.1\right)$.

\section{Controlling for structured noise}

Our preprocessing stream included several steps to limit the influence of structured noise, such as motion artifacts [55], heartbeat [52], and respiration [53]. First, we conducted manual ICA-based artifact removal. The first author visually inspected all the independent component maps for each participant to identify noise components based on the spatial layout of the component maps and the power spectra of the associated time series [56]. We applied nonaggressive denoising with FSL's fsl_regfilt, i.e., only variance that was uniquely related to the components labeled as noise component (approx. $70 \%$ ) was removed.

After ICA-based noise removal and further preprocessing, we conducted nuisance regression modeling the potential effect from motion and physiological noise on the resting state fMRI data. Specifically, we included six rigid body parameters and the eigenvariate of signals over the entire white matter and the cerebrospinal fluid (CSF) in our GLM. Moreover, we calculated 10 cardiac phase regressors, 10 respiratory phase regressors, and 6 other nuisance regressors including heart rate fluctuation (HRF), heart rate variability (HRV), respiration raw data averaged per TR, respiratory amplitude in 9-s window, respiratory frequency in 9-s window, and RVT (frequency times amplitude of respiration, averaged per TR) that are derived from retrospective image correction (RETROICOR) method [57].

\section{Region of interest selection}

Stereotaxic, probabilistic maps of the cytoarchitectonic Juelich histological atlas distributed along with FSL were created for the LB (left, $1032 \mathrm{~mm}^{3}$; right, $928 \mathrm{~mm}^{3}$ ), centromedial (CM) (left, $16 \mathrm{~mm}^{3}$; right, $40 \mathrm{~mm}^{3}$ ), and superficial (SF) (left, $400 \mathrm{~mm}^{3}$; right, $160 \mathrm{~mm}^{3}$ ) nuclei (Fig. 1a). The CM part includes the central and medial subdivision. The LB compartment comprises the lateral, basolateral, basomedial, and paralaminar nuclei. The SF 


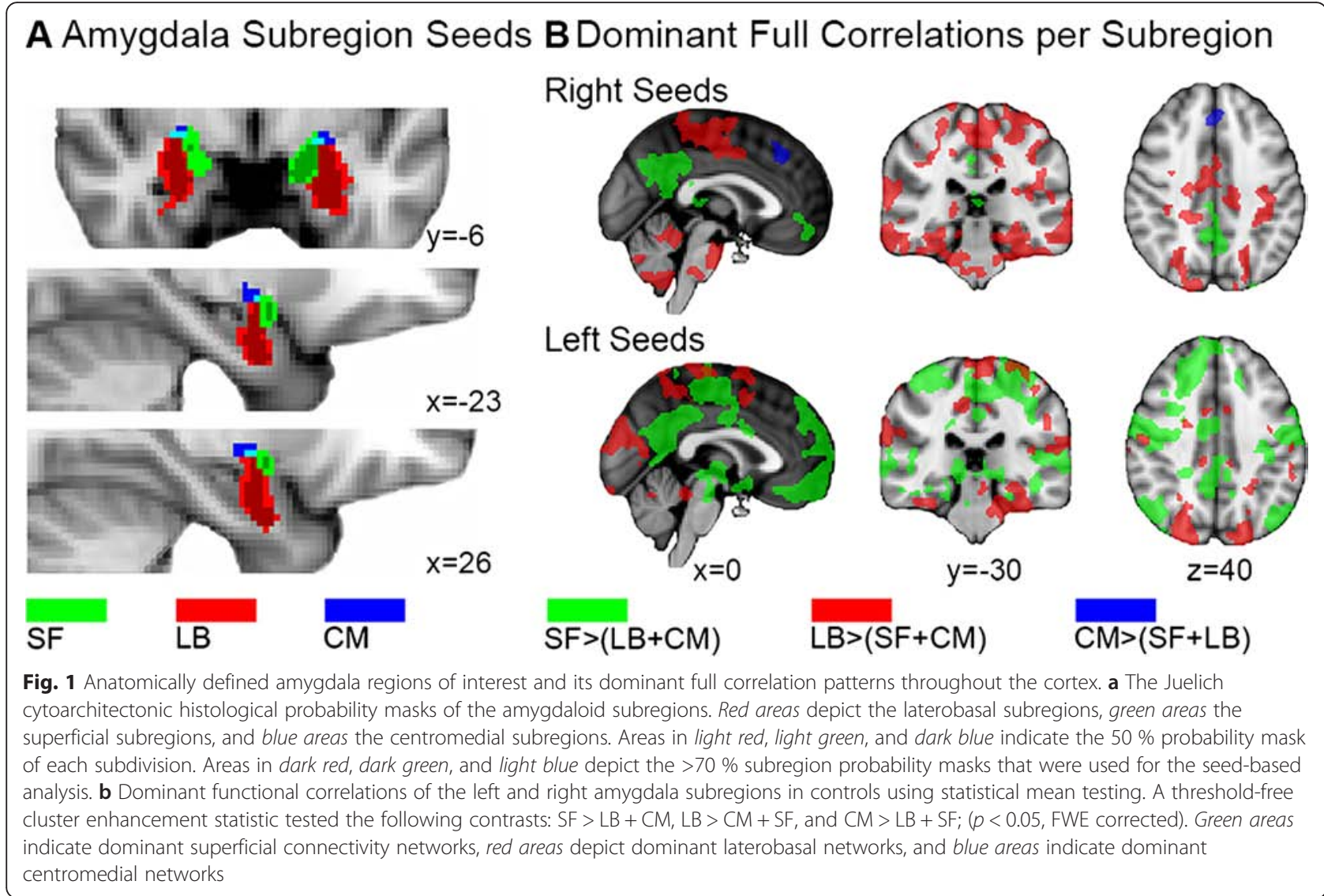

subcompartment incorporates the anterior amygdaloid area, the amygdalopyriform transition area, the amygdaloid-hippocampal area, and the ventral and posterior cortical nuclei. Only voxels with a greater than $70 \%$ probability to represent the respective subregion were included in the analysis to reduce overlap between subregions. The entire amygdala region of interest (ROI) was constructed by combining the three amygdaloid subregions into a single structure.

\section{Statistical analysis}

First level analyses were carried out using FSL's seedbased correlation analysis (SBCA) [58] to calculate the partial correlation between the average time series of the voxels in one ROI (i.e., one of the three amygdala subregions) and the time series of every voxel of the whole brain, corrected for the average time series of the other two amygdala seeds. This tool incorporates the option to add data with different smoothing kernels for the seed and target areas. For the amygdala, cortical correlation analysis we entered spatially unfiltered data for the small amygdaloid areas and FWHM $=6 \mathrm{~mm}$ Gaussian filtered functional images for the whole brain. Thus, one singlesubject partial correlation map of the brain for each subregion (left and right CM, LB, and SF) was obtained, yielding each subregion's unique connectivity with the rest of the brain. In addition to the partial correlation analyses, we also performed a regular correlation analysis of the entire amygdala (EA) (left and right) with every voxel in the brain to serve as a reference for the partial correlation results, increasing the interpretability of the partial correlation results.

To test for between-group differences, we performed a nonparametric test with Randomise [59]. As this analysis does not require the data to be normally distributed, an $\mathrm{r}$ to $\mathrm{z}$ transform is not necessary. Thus, 5000 random permutations of a threshold-free cluster enhancement statistic (TFCE) [60] against the null hypothesis were conducted for each ROI separately. The $p$ values were extracted with FSL's cluster command, where cluster peaks and local maxima with $p<0.05$ were acquired from the threshold-free cluster enhancement FWE corrected 1-p statistical images of the Randomise output. The permutation method strongly controls for the family-wise error (FWE) rate when a large amount of voxels is tested. Six contrasts (positive main effects in ASD; positive main effects in controls, negative main effects in ASD; negative main effects in controls; ASD > controls; controls > ASD) were tested with an unpaired samples $t$ test. The same approach was used for entire amygdala analysis. 
The demographic data (IQ, age, and AQ) of both experimental groups were compared using analysis of variance (ANOVA) in SPSS 20 [61] (Table 1).

\section{Results}

\section{Entire amygdala connectivity}

To obtain a reference analysis for the subregion-specific amygdala approach and to compare our results with previous literature, we first mapped the intrinsic connectivity of the entire amygdala (Fig. 2a). Only significant clusters surviving family-wise error correction with alpha $<0.05$ are reported (Additional file 2).

Figure 2a presents the positive entire amygdala correlations in the ASD and control sample. This reveals a number of symmetric left and right frontal, occipital, temporal, and sensorimotor networks including the cingulate gyrus, which is consistent with the amygdala's role in socio-emotive circuits [62]. Left amygdala correlations yielded one large cluster with its peak in the left anterior parahippocampal gyrus $(-28,-8,-36 ; p<0.001)$.
Local maxima extended dorsally into the left precentral gyrus, superior parietal lobe, and both sides of the postcentral gyrus and ventrally into the ipsilateral superior temporal gyrus, planum temporale, and temporal pole. Right amygdala correlations were found in the anterior and posterior cingulate gyrus $(0,-2,44 ; p=0.012)$, including the supplementary motor cortex and clusters in the right temporal fusiform cortex $(32,-6,-36 ; p<0.001)$, middle temporal gyrus, central opercular cortex, supramarginal gyrus, parietal operculum cortex, a cluster in the left superior temporal gyrus $(-62,-8,-4 ; p=0.002)$, and a number of smaller clusters in the left inferior temporal gyrus $(-46,-58,-16 ; p=0.036)$.

The results of the ASD group are comparable with the control group's results with symmetric left and right frontal, occipital, temporal, and sensorimotor networks underlying emotion regulation circuits (see EA patients in Fig. 2a). The left amygdala revealed a large cluster in the left temporal pole $(-26,4,-46 ; p<0.001)$ with local maxima in the bilateral pre- and postcentral gyrus. The

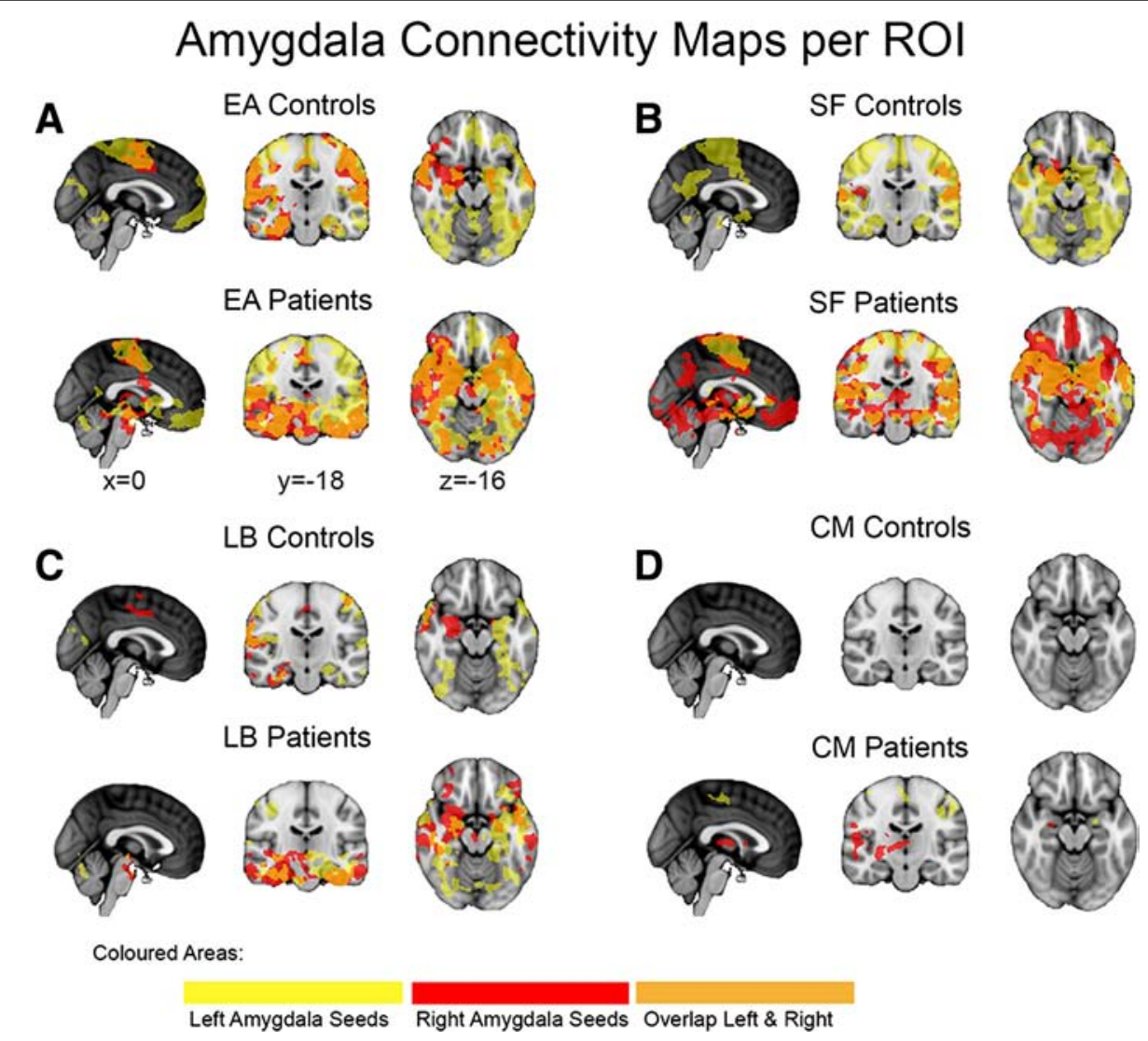

Fig. 2 Intrinsic positive connectivity networks of the entire amygdala and its individual subregions in controls and patients. a Significant results ( $p<0.05$ FWE corrected) of entire amygdalo-cortical full correlation analyses are delineated for the ASD group (EA patients) and controls (EA controls). Yellow and red areas depict results from the left and right amygdala seeds, respectively, with orange regions illustrating its overlap. Positive main effects ( $p<0.05$ FWE corrected) of the subregion-specific correlation analyses are shown in the same color code for the $\mathbf{b}$ superficial amygdala in patients (SF patients) and controls (SF controls), $\mathbf{c}$ the laterobasal amygdala in patients (LB patients) and controls (LB controls) and d the centromedial amygdala in patients (CM patients) and controls (CM controls) 
right amygdala yielded a large cluster in the right temporal pole $(28,6,-42 ; p<0.001)$ including the local maxima in the temporal areas, the brainstem, and the right hippocampus.

Negative correlations were largely absent in our sample. This is probably a consequence of (1) the participants' age because during adolescence, negative (inhibitory) mechanisms are not yet as exuberant as after puberty's transition phase [63-65] and (2) no global signal regression was performed, which could have introduced spurious negative correlations $[66,67]$ in previous work that did subtract the global mean signal.

\section{Subregion-specific connectivity}

Next, to investigate the individual contributions of the nuclei group-specific seeds, we mapped the intrinsic whole brain connectivity of each ROI (Fig. 2b-d). As before, only significant clusters surviving family-wise error correction with alpha $<0.05$ are reported (Additional files $3,4,5$, and 6).

\section{Superficial amygdala connectivity}

Left SF connectivity in controls (SF controls, yellow) showed extensive unique bilateral positive correlations throughout frontal (right frontal medial cortex $(4,44,-20$; $p=0.029)$, left frontal pole $(-2,62,-6 ; p=0.031)$, temporal (left parahippocampal gyrus $(-14,-6,-26 ; p<$ 0.001 ), occipital, and parietal lobe and limbic areas including anterior and posterior cingulate gyrus, parahippocampal gyrus, hippocampus, amygdala, thalamus, putamen as well as in the brainstem and cerebellum (Additional files 3 and 6). The right SF showed connectivity to smaller, more selective sensory and limbic areas. In ASD (Fig. 2b, SF patients), SF connectivity yielded a similar pattern, except for a larger cluster in the frontal lobe area, with sparse overall occipital lobe connectivity.

Note that while partial correlation analysis reported for the SF yielded a relatively small frontal lobe cluster in our healthy subjects, a supplementary full correlation analysis revealed a large frontal lobe involvement, indicating that the overlapping signal between SF and $\mathrm{CM} / \mathrm{LB}$ time series leads to partialling out some of the SF-frontal connectivity (Fig. 1b). Thus, the results indicate that (1) SF functional connectivity in our adolescent sample is consistent with known limbic-striatal-frontal reward related and auditory-parietal-visual valence evaluating circuits [68] and (2) the SF subregion maintains unique whole brain connectivity.

\section{Laterobasal amygdala connectivity}

The laterobasal subregion exhibited mainly unique connectivity with temporal regions and regions along the lateral superior cortical axis. Although left and right LB connectivity maps overlapped in the parietal operculum cortex, frontal orbital cortex, and temporal pole, strong lateralization effects were also observed (Fig. 2c, LB controls). Left LB connectivity peaks were bilaterally present in the parahippocampal gyrus (left $(-26,-8,-34 ; p<0.001)$; right $(32,-28,-30 ; \quad p=0.047)$ ), precentral gyrus (left $(-44,-14,56 ; p=0.014))$; right $(42,-14,52 ; p=0.017))$, and lateral occipital cortex (left $(-20,-80,42 ; p=0.020)$; right $(30,-84,28 ; p=0.021)$ ) (Additional file 4). Except from one large cluster of connectivity in the right temporal fusiform cortex $(32,-6,-36 ; p<0.001)$, right LB showed many localized clusters of connectivity, such as in the right supplementary motor area $(2,-8,62 ; p=0.033)$, right frontal orbital cortex $(44,28,-18 ; p=0.048)$ and bilateral anterior cingulate gyrus (left $(6,-12,44 ; p=0.031)$; right $(0,-2,44 ; p=0.033))$. In the ASD group, similar patterns of connectivity were observed (Fig. 2c, LB patients). Thus, the LB connectivity maps are in line with its putative involvement in emotional learning through its connection with the (para)hippocampal area and its involvement in multisensory processing via its projections to the sensory systems along the superior temporal gyrus, sensorimotor areas, and visual areas, in combination with cingulate-frontal connectivity [69].

\section{Centromedial amygdala connectivity}

In the control group, only a small cluster in the right occipital fusiform gyrus $(28,-64,-10 ; p=0.040)$ from the right centromedial subregion showed a negative correlation, while positive unique functional connectivity did not reach the significance threshold for the left or right $\mathrm{CM}$ in the control group using partial correlation analysis (Fig. 2c, CM controls). We therefore performed an additional, regular correlation analysis for the $\mathrm{CM}$ to test whether some of its unique signal was "partialled out" due to a functional overlap between CM and LB/SF nuclei (Fig. 1b). As expected, and in line with previous reports [37], positive correlations in anterior cingulate cortex were significantly higher for the CM nucleus compared to SF and LB in healthy controls. This suggests that the partial correlation approach had corrected for the strong overlap with time series from LB and SF to such an extent that the CM's unique contribution did not reach the FWEcorrected threshold (Additional file 7). The relative lack of unique $\mathrm{CM}$ correlations in controls may well reflect the lower degree of functional specialization of the amygdaloid nuclei in adolescents [70]. In the ASD group, however, left $\mathrm{CM}$ demonstrated unique partial correlations with bilateral primary sensorimotor areas (postcentral gyrus (left, $-36,-22,40 ; p=0.004$ ), precentral gyrus (right, $40,-14,60 ; p=0.007)$ ), and left insular cortex in the partial correlation analysis, while right CM correlations with the striatum (including bilateral thalamus, left putamen $(-24,0,-10 ; p=0.046)$ and right pallidum) and right 
hemispheric speech processing areas (including central and frontal opercular cortex, supramarginal gyrus, and Heschl's gyrus) were present (Fig. 2c, CM patients; Additional file 5).

\section{Difference between ASD and controls Entire amygdala connectivity}

In line with our hypothesis, we found significantly smaller left amygdala correlations with the left hemispheric occipital pole $(-24,-90,34 ; p=0.026)$, supracalcarine cortex and intracalcarine cortex, left $(-26,-60,58$; $p=0.040)$ and right lateral occipital cortex $(28,-58,64$; $p=0.049)$ in ASD compared to controls. Furthermore, correlations between left amygdala and cuneal cortex were reduced in both hemispheres in ASD. The right amygdala showed significantly reduced correlations with the right superior parietal lobe $(14,-54,62 ; p=0.023)$ in ASD (Fig. 3a; Additional file 8).

\section{Subregion-specific connectivity}

To test our hypothesis of reduced amygdala connectivity along sensory input channels in ASD, we directly compared the SF, LB, and CM connectivity between groups (Fig. 3b; Additional file 8). In line with our hypothesis, the ASD group showed reduced left SF connectivity with bilateral precuneus cortex (left, 16,-58,8; $p=0.003$ ), cuneal cortex, intracalcarine cortex, lateral occipital cortex (right, 44,-60,12; $p=0.023$ ), and supracalcarine cortex in the right hemisphere, the occipital pole, the superior parietal lobe $(28,-54,62 ; p=0.004)$, and the preand postcentral and angular gyri $(44,-16,64 ; p=0.047)$ when compared with controls. Furthermore, functional connectivity between the right LB and right superior parietal lobe $(14,-54,62 ; p=0.023)$ were reduced in ASD. There were no significant between-group differences in the CM correlation maps. Statistical tests in both directions (ASD > controls; controls > ASD) were included in our analysis, but yielded nonsignificant

\section{Reduced FC: Patients $<$ Controls}

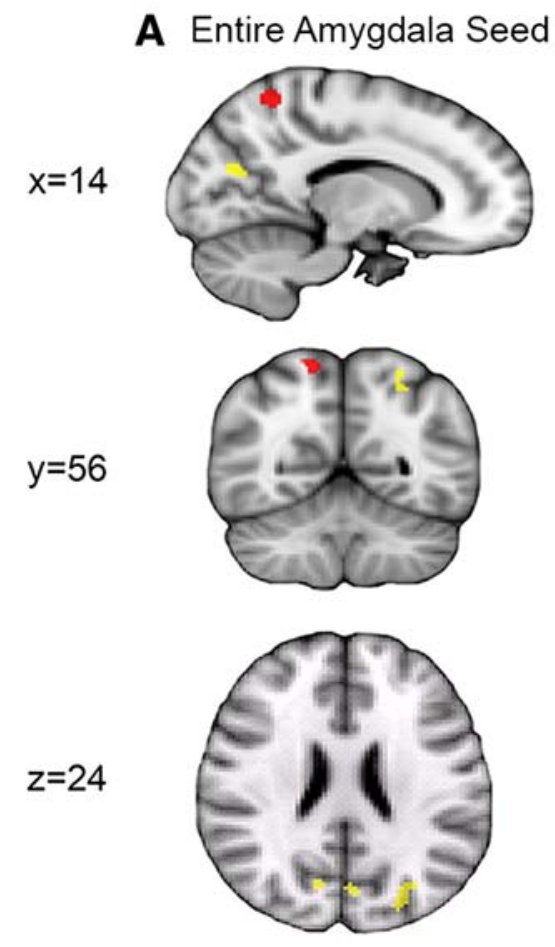

Left EA Right EA
B Subregion Seed
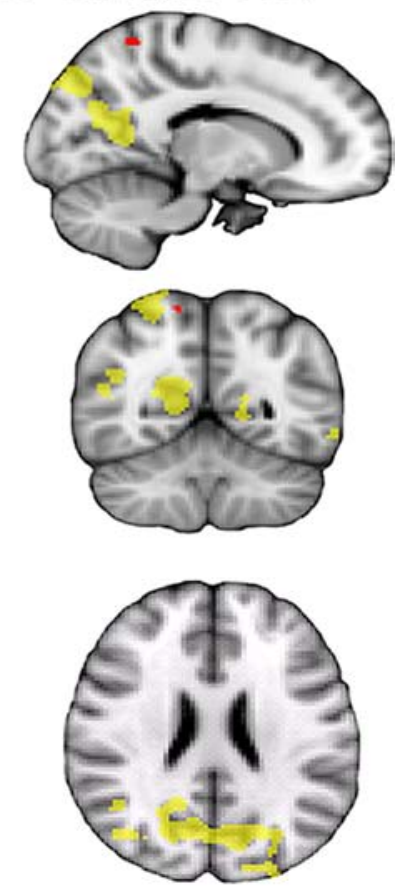

Left SF Right LB

Fig. 3 Areas of reduced functional connectivity in ASD. a Significant ( $p<0.05$; FWE corrected) reduced connectivity with the entire amygdala (EA) ROI. Yellow areas show between-group differences in connectivity with left amygdala seeds, while red areas show connectivity with right amygdala seeds. $\mathbf{b}$ Conventions are depicted as in panel (a) but with yellow regions illustrating the left superficial ROI and red indicating the right laterobasal subcompartment. The results from the partial correlation analysis revealed that the between-group difference in EA was driven by the left SF and right LB. Bilateral CM, right SF, and left LB did not yield significant between-group differences 
results for ASD overconnectivity patterns. When the analysis was repeated including age as covariate, the group effects were very similar to the previous betweengroup results and no age-by-group interactions were found. Age showed a negative main effect with connectivity strength in the left SF, indicating that connectivity strength decreases during development in the temporal pole and lateral occipital cortex (Additional file 9).

Relation between reduced connectivity strength and AQ scores To investigate whether the subregionspecific group differences in functional connectivity can be related to specific behavioral/symptomatic characteristics, we quantified the relationship between the participants' scores within each group on each AQ subdomain and the (z-transformed) correlation between the mean fMRI time series from the right LB and left SF nuclei and the cortical regions that exhibited a significant group difference in functional connectivity. For either group, however, none of the specific AQ sub-domains were significantly related to the subregion-specific functional connectivity strengths. This absence of significance was also observed for the relationship between total AQ score and subregion-specific connectivity strength.

\section{Subregion signal-to-noise ratios}

In theory, the differences in size in the amygdaloid nuclei seed regions might have induced different tSNR (time series' signal-to-noise ratios) levels between seed regions. To rule out this possibility, we tested whether tSNR varied significantly across seed regions using subject-wise tSNRs from the preprocessed functional images before ICA denoising (Additional file 10). A threeway ANOVA (factors: subregion, diagnostic group, and hemisphere) demonstrated that tSNRs differed between hemispheres $(F=8.724, d f=1, p=0.003)$ and diagnostic groups $(F=3.770, d f=1, p=0.53)$, but not between subregions $(F=0.348, d f=2, p=0.707)$. Furthermore, there were no significant interaction effects between subregions, diagnostic groups, and hemispheres, indicating that differences in subregion tSNR were not affected by diagnostic status or lateralization effects (Table 2). Therefore, the absence of CM main effects in the control group as well as its negative findings in the betweengroup analysis is not likely caused by different tSNR levels of the CM subregion.

\section{Discussion}

In the present study, we investigated amygdalo-cortical connectivity in adolescents and young adults with ASD and controls during resting state fMRI. We found reduced cortical connectivity of both amygdaloid input subregions (SF and LB) with the prefrontal, parietal, and occipital cortices in participants with ASD, while CM output connectivity was spared.

The positive correlation maps from both the entire amygdala and the three subregions in our control group of healthy adolescents revealed large overall overlap with the spontaneous activation maps previously reported in healthy adults [33]. The present results indicate that most of the entire amygdala connectivity main effects can be disentangled into subunit functionality in adolescents and young adults with and without ASD, while some of the global effects (e.g., frontal lobe activation) could not be traced down to one particular subunit. As positive partial CM correlations did not reach FWEcorrected significance in the control group, one interpretation of these findings could entail a lower degree of functional specialization in healthy adolescents. While this may reflect the not yet fully differentiated amygdala in adolescence, the conservative partial correlation approach may also have contributed to the negative finding because normal CM anterior cingulated gyrus connections were found with the full correlation approach. The absence of significant partial correlations for the $\mathrm{CM}$ compartment in the controls is most likely due to commonalities (i.e., shared variance) between the $\mathrm{CM}$ signals and those from the other subregions, leading them to be partialled out. Indeed, a direct comparison revealed no significant differences between patients and controls. Positive laterobasal-cortical correlations in the prefrontal, parietal, and temporal cortex further confirmed

Table 2 Analysis of subregion signal-to-noise ratios

\begin{tabular}{llccc}
\hline Factor & $d f$ & Mean square & $F$ & $p$ value \\
\hline Hemisphere & 1 & 301.406 & 8.724 & $0.003^{*}$ \\
Subregion & 2 & 12.014 & 0.348 & 0.707 \\
Diagnostic group & 1 & 130.24 & 3.770 & 0.053 \\
Hemipshere*Subregion & 2 & 18.722 & 0.542 & 0.582 \\
Subregion*Diagnostic group & 2 & 1.031 & 0.030 & 0.971 \\
Hemisphere*Diagnostic group*Subregion & 3 & 10.098 & 0.292 & 0.831
\end{tabular}

$p$ values indicate results for between-subjects effects of tSNR (time-series' signal-to-noise ratios), $d f$ degrees of freedom, $F$ univariate ANOVA

${ }^{*}$ statistically significant 
previous findings that connect the LB with associative learning processes across sensory modalities [33, 71], while SF-cortical correlations were in line with known limbic lobe connectivity [33].

Importantly, we found reduced EA connectivity in the ASD group when compared to controls. In line with our hypothesis of amygdala underconnectivity in ASD, we found reduced left EA connectivity with portions of the occipital pole, cuneal cortex, intraand supracalcarine cortex and lateral occipital cortex in the ASD group. Partial correlation analysis revealed that these differences were largely driven by the left SF subregion, with reduced left SF connectivity in the precuneus, cuneus, angular gyrus, precentral, and postcentral gyrus and superior parietal cortex. The ASD group also exhibited reduced right entire amygdala connectivity with the right superior parietal lobe. Partial correlation analysis revealed that this difference was mainly driven by the right LB.

Prior research showed that functional specialization of amygdaloid subregions continues throughout adolescence $[70,72]$ and that volumetric abnormalities in ASD are age specific $[10,11]$. The possibility that our between-group effects were driven by developmental differences was however not supported by our data. There were no groupby-age interactions for EA and subregion-specific connectivity, and our reported between-group effects were similar to the between-group effects with age as covariate. The negative main effects of age showed no overlap with the abnormalities found for the left SF in our ASD group. Thus, while the age effect in the left superficial area might reflect the developmental changes of the amygdaloid subcompartments throughout adolescence as reported by Gabard-Durnam and colleagues [72] and Qin et al. [70], the amygdala-cortical abnormalities in ASD are not significantly age dependent, at least within the age range examined in the present study.

Although our amygdalo-cortical analysis was not specifically designed for investigating local overconnectivity, increased activation patterns in ASD were also tested and yielded nonsignificant results. Investigating the local overconnectivity account remains challenging in fMRI research [73], and only few studies particularly investigated local overconnectivity in fMRI resting state $[19,21-23]$ and generated inconsistent results.

Previous task-based fMRI studies have reported some support for pathway-specific deficits of the parietal visuospatial domain in ASD $[28,29,74]$ and its connections to the amygdala [75]. Since reduced functional amygdalo-cortical connectivity in our ASD sample was mainly present in the dorso-dorsal and ventro-dorsal pathway, our results suggest that abnormal amygdaloid connectivity in ASD is pathway specific. That is, the fact that we only found abnormal connectivity with the left $\mathrm{SF}$ and the right LB, i.e., the amygdaloid input areas, but not with the CM output areas, supports the notion that social-emotional deficits in ASD may be reflected in reduced connectivity along amygdalo-sensory input pathways. Thus, a deficiency specific to the amygdalocortical input pathway may account for the social perceptual deficits in ASD.

Our results also showed lateralized subregion-specific amygdaloid connectivity, which contradicts a previous finding of bilateral homogenous amygdala connectivity [33]. However, a number of studies associated the left amygdala with slower explicit emotion appraisal processes, while the right amygdala is more involved with faster implicit threat detection [76]. Another hemispheric lateralization account distinguishes the left hemispheric abstract category subsystem and the right hemispheric whole-based subsystem: the left subsystem uses a parts-based processing strategy to represent smaller features of larger whole objects, while the right hemispheric whole-based subsystem serves visual discrimination of similar objects (see [77]). As the amygdalo-cortical deficits in our ASD sample accumulated along the left SF and right LB sensory input pathways, while other amygdalo-cortical pathways were spared, the results might indicate that the cortical processing of visual object features may be affected in ASD. That is, deficits in the left SF may account for abnormal parts-based perceptual processes along the ventro-dorsal and dorso-dorsal perceptual pathway, while abnormal functional connectivity in the right LB might for instance reflect whole-object face processing difficulties in ASD caused by abnormal amygdalo-cortical connectivity with the right superior parietal lobe.

Within groups, we did not observe a significant relationship between the subregion-specific reductions in functional connectivity strength and the participants' AQ scores. Given the observed differences in subregionspecific functional connectivity strength across groups, and the fact that ASD status and AQ score are clearly related (see Table 1), it is likely that the absence of statistical significance for these within-group comparisons is related to insufficient statistical power. Future work based on larger sample sizes may therefore be able to tease apart the effects of subregion-specific reductions in functional connectivity on behavior.

Our investigative approach draws on Roy and colleagues' paper that describes resting state analysis of probabilistic cytoarchitectonically defined amygdala nuclei [33]. In our study, however, partial correlation analysis was used instead of regression analysis with statistical mean testing to compute the unique contributions of each of the three amygdaloid subdivisions, and we studied adolescent brains rather than healthy adult 
brains. As such, small differences between the two studies may be expected. For instance, in our study, the CM subcompartment showed connectivity with the striatal circuitry in the ASD group, while positive partial correlations did not reach FWE-corrected significance in the control group. Because we did find normal positive correlations in a full correlation analysis for the CM (Fig. 1b), we interpret the lack of partial CM-wholebrain correlations in controls as a reflection of the lower degree of functional specialization of the amygdaloid nuclei in the adolescent brain [70]. Further support for the notion of functional overlap between our three subcompartments was found during supplementary analysis of colinearity (Additional file 7). A putative faster maturation of the CM region in ASD as compared to controls was not supported by the data; we directly tested for increased connectivity patterns in ASD compared to controls but found no difference. Overall, we regard our method as a valid approach for detecting true functional connectivity differences between healthy and clinical populations [78], since (1) the entire amygdala results showed strong overlap with the subregionspecific outcomes from the partial correlation analysis (Additional file 11), (2) the current results clearly demonstrate the expected functional connectivity with known amygdala circuits, and (3) sensitivity to betweengroup effects increases with partial correlation analysis (Fig. 3, Additional file 8).

\section{Conclusions}

To conclude, the current findings provide further evidence for underconnectivity in socio-emotive circuits in adolescents and young adults with ASD. As we found abnormal connectivity in the amygdala's input areas but not in the output areas, the findings support the notion that deficient/impaired amygdaloid sensory input mechanisms may underlie ASD. This might indicate that therapeutic interventions should target sensory input channels.

\section{Additional files}

Additional file 1: Relative frame-wise displacement. Indicates movement between fMRI scans in participants with autism spectrum disorder and control subjects. (DOC $41 \mathrm{~kb}$ )

Additional file 2: Intrinsic entire amygdalo-cortical functional connectivity. Demonstrates main effects of left entire and right entire amygdalo-cortical correlation analysis in participants with autism spectrum disorder and control subjects. (DOC $228 \mathrm{~kb}$ )

Additional file 3: Intrinsic superficial-cortical functional connectivity. Demonstrates main effects of left and right superficialcortical partial correlation analysis in participants with Autism Spectrum Disorder and control subjects. (DOC $196 \mathrm{~kb}$ )

Additional file 4: Intrinsic laterobasal-cortical functional connectivity. Demonstrates main effects of left and right laterobasal- cortical partial correlation analysis in participants with autism spectrum disorder and control subjects. (DOCX $384 \mathrm{~kb}$ )

Additional file 5: Intrinsic centromedial-cortical functional connectivity. Demonstrates main effects of left and right centromedialcortical partial correlation analysis in participants with autism spectrum disorder and control subjects. (DOCX 109 kb)

Additional file 6: Intrinsic amygdalo-cortical functional connectivity in superficial subregion. Demonstrates a selection of superficial-cortical partial correlation main effects with adjusted significant thresholds in participants with autism spectrum disorder and control subjects. (DOC 253 kb)

Additional file 7: Functional overlap between the superficial, laterobasal, and centromedial subregions in control subjects. (DOC $30 \mathrm{~kb}$ )

Additional file 8: Reduced functional connectivity in ASD. Demonstrates between-group differences of entire amygdalo-cortical correlations and subregion-specific partial correlations between participants with autism spectrum disorder and control subjects. (DOC 107 kb)

Additional file 9: Functional connectivity with age effects. Demonstrates between-group differences of entire amygdalo-cortical correlations and nuclei group-specific partial correlations between participants with autism spectrum disorder and control subjects and negative main effects of age in the superficial subcompartment. (DOC $85 \mathrm{~kb}$ )

Additional file 10: Subject-wise tSNR levels per nucleus group. (DOC $4303 \mathrm{~kb}$ )

Additional file 11: Similarity between all partial correlation maps combined and entire amygdala correlation maps in healthy subjects. (DOC $55 \mathrm{~kb}$ )

\section{Abbreviations}

ABCL/18-59: Adult Behavior Check List/age 18-59; ADI-R: Autism Diagnostic Interview-Revised; ADHD: attention deficit/hyperactivity disorder; ANOVA: analysis of variance; ASD: autism spectrum disorder; AQ: autism spectrum quotient; BBR: boundary-based registration; BET: Brain Extraction Tool; BOLD: blood-oxygen-level dependent; BO: symbol for the constant magnetic field; CBCL/6-18: Child Behavior Check List/age 6-18;

CM: centromedial amygdaloid subcompartment; CSF: cerebrospinal fluid; DSM-4: Diagnostic and Statistical Manual of Mental Disorders (4th edition); DTI: diffusion tensor imaging; EA: entire amygdala; EPI: echo-planar imaging; FC: Functional Connectivity; FD: frame-wise displacement; fMRI: functional magnetic resonance imaging; FMRIB: (Oxford centre for) functional magnetic resonance imaging of the brain; FNIRT: FMRIB's nonlinear image registration tool; FOV: field of view, spatial encoding area of the image; FSL: FMRIB Software Library; FUGUE: FMRIB's Utility for Geometrically Unwarping EPIs; FWHM: full width at half maximum; HRF: heart rate fluctuation; HRV: heart rate variability; ICA: independent component analysis; IQ: intelligence quotient; LB: laterobasal amygdaloid subcompartment; MCFLIRT: FMRIB's Linear registration and motion correction tool; MELODIC: Multivariate Exploratory Linear Optimized Decomposition into Independent Components; MPRAGE: magnetization-prepared rapid acquisition with gradient echo; MRI: magnetic resonance imaging; PRELUDE: Phase Region Expanding Labeller for Unwrapping Discrete Estimates; RETROICOR: retrospective image correction; ROI: region of interest; RVT: respiration volume per time; SBCA: seed-based correlation analysis; SF: superficial amygdaloid subcompartment; SPSS 20: Statistical Package for the Social Sciences (20th edition); TE: echo time; Tl: inversion time; TR: repetition time; T1-weighted: an image whose contrast and brightness are predominately determined by $\mathrm{T} 1$ signals (T1: time constant at which spins realign themselves with the external magnetic field after excitation); T2*: T-two-star, time constant for the loss of phase coherence among spins oriented at an angle to the static magnetic field; WAIS-III: Wechsler Adult Intelligence Scale (3rd edition); WISCIII: Wechsler Intelligence Scale for Children (3rd edition).

\section{Competing interests}

The authors declare that they have no competing interests. 


\section{Authors' contributions}

$A R, C B, E O, K H, M M, W G$, and WZ contributed to data-analysis. CB, EH, GvW $J B, K H$, and WG designed the study. AR, EO, WZ, and WG acquired the data. All authors contributed to the drafts of the manuscript and approved the final version.

\section{Acknowledgements}

This work was supported by the Hersenstichting Fellowship (F2010(1)-13). The authors would like to thank Marieke W.M. Kuiper for her assistance with the data collection.

\section{Author details}

'Department of Cognitive Neuroscience, Radboud University Medical Center Nijmegen, P.O. Box 91016500 HB Nijmegen, The Netherlands. ${ }^{2}$ Donders Institute for Brain, Cognition and Behaviour, Centre for Cognitive Neuroimaging, Radboud University, Nijmegen, The Netherlands. ${ }^{3}$ MIRA Institute, University of Twente, Enschede, The Netherlands. ${ }^{4}$ Department of Psychiatry, Academic Medical Center, University of Amsterdam, Amsterdam, The Netherlands. ${ }^{5}$ Centre for Functional MRI of the Brain (FMRIB), University of Oxford, Oxford, United Kingdom. ${ }^{6}$ Karakter Child and Adolescent Psychiatry University Centre, Nijmegen, The Netherlands.

Received: 10 April 2015 Accepted: 7 December 2015

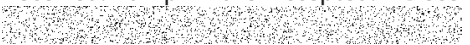

\section{References}

1. American Psychiatric Association. Diagnostic and statistical manual of mental disorders: DSM-IV-TR ${ }^{\oplus}$. Washington (DC): American Psychiatric Publisher; 2000.

2. American Psychiatric Association. Diagnostic and statistical manual of mental disorders. 5th ed. Washington (DC): American Psychiatric Publisher; 2013.

3. Verhoeven JS, De Cock P, Lagae L, Sunaert S. Neuroimaging of autism. Neuroradiology. 2010;52(1):3-14.

4. Just MA, Cherkassky VL, Keller TA, Kana RK, Minshew NJ. Functional and anatomical cortical underconnectivity in autism: evidence from an FMRI study of an executive function task and corpus callosum morphometry. Cereb Cortex. 2007;17(4):951-61.

5. Anagnostou E, Taylor MJ. Review of neuroimaging in autism spectrum disorders: what have we learned and where we go from here. Mol Autism. 2011;2:4.

6. Amaral DG, Schumann CM, Nordahl CW. Neuroanatomy of autism. Trends Neurosci. 2008;31(3):137-45.

7. Ashwin C, Chapman E, Colle L, Baron-Cohen S. Impaired recognition of negative basic emotions in autism: a test of the amygdala theory. Soc Neurosci. 2006;1(3-4):349-63.

8. Baron-Cohen S, Ring HA, Bullmore ET, Wheelwright S, Ashwin C, Williams SC. The amygdala theory of autism. Neurosci Biobehav Rev. 2000;24(3):355-64.

9. Phelps EA, LeDoux JE. Contributions of the amygdala to emotion processing: from animal models to human behavior. Neuron. 2005;48(2):175.

10. Bellani M, Calderoni S, Muratori F, Brambilla P. Brain anatomy of autism spectrum disorders II. Focus on amygdala. Epidemiol Psychiatr Sci. 2013;22:309-12.

11. Groen W, Teluij M, Buitelaar J, Tendolkar I. Amygdala and hippocampus enlargement during adolescence in autism. J Am Acad Child Adolesc Psychiatry. 2010;49(6):552-60.

12. Green SA, Rudie JD, Colich NL, Wood JJ, Shirinyan D, Hernandez L, et al. Overreactive brain responses to sensory stimuli in youth with autism spectrum disorders. J Am Acad Child Adolesc Psychiatry. 2013;52(11):1158-72.

13. Tottenham N, Hertzig ME, Gillespie-Lynch K, Gilhooly T, Millner AJ, Casey B. Elevated amygdala response to faces and gaze aversion in autism spectrum disorder. Soc Cogn Affect Neurosci. 2014;9(1):106-17.

14. Nacewicz BM, Dalton KM, Johnstone T, Long MT, McAuliff EM, Oakes TR, et al. Amygdala volume and nonverbal social impairment in adolescent and adult males with autism. Arch Gen Psychiatry. 2006;63(12):1417-28.

15. Schumann CM, Barnes CC, Lord C, Courchesne E. Amygdala enlargement in toddlers with autism related to severity of social and communication impairments. Biol Psychiatry. 2009;66(10):942-9.

16. Courchesne E, Pierce K. Why the frontal cortex in autism might be talking only to itself: local over-connectivity but long-distance disconnection. Curr Opin Neurobiol. 2005;15(2):225-30.

17. Anderson JS, Druzgal TJ, Froehlich A, DuBray MB, Lange N, Alexander AL, et al. Decreased interhemispheric functional connectivity in autism. Cereb Cortex. 2011;21(5):1134-46.
18. Travers BG, Adluru N, Ennis C, Tromp DP, Destiche D, Doran S, et al. Diffusion tensor imaging in autism spectrum disorder: a review. Autism Res. 2012;5(5):289-313.

19. Di Martino A, Zuo X-N, Kelly C, Grzadzinski R, Mennes M, Schvarcz A, et al. Shared and distinct intrinsic functional network centrality in autism and attention-deficit/hyperactivity disorder. Biol Psychiatry. 2013;74(8): 623-32.

20. Khan S, Gramfort A, Shetty NR, Kitzbichler MG, Ganesan S, Moran JM, et al. Local and long-range functional connectivity is reduced in concert in autism spectrum disorders. Proc Natl Acad Sci U S A. 2013;110(8):3107-12.

21. Maximo JO, Keown CL, Nair A, Müller R-A. Approaches to local connectivity in autism using resting state functional connectivity MRI. Front Hum Neurosci. 2013;7:605

22. Paakki J-J, Rahko J, Long X, Moilanen I, Tervonen O, Nikkinen J, et al. Alterations in regional homogeneity of resting-state brain activity in autism spectrum disorders. Brain Res. 2010;1321:169-79.

23. Shukla DK, Keehn B, Müller RA. Regional homogeneity of fMRI time series in autism spectrum disorders. Neurosci Lett. 2010;476(1):46-51.

24. Mottron L, Dawson M, Soulieres I, Hubert B, Burack J. Enhanced perceptual functioning in autism: an update, and eight principles of autistic perception. J Autism Dev Disord. 2006;36(1):27-43.

25. Samson F, Mottron L, Jemel B, Belin P, Ciocca V. Can spectro-temporal complexity explain the autistic pattern of performance on auditory tasks? J Autism Dev Disord. 2006;36(1):65-76.

26. Groen WB, van Orsouw L, Ter Huurne N, Swinkels S, van der Gaag R-J, Buitelaar JK, et al. Intact spectral but abnormal temporal processing of auditory stimuli in autism. J Autism Dev Disord. 2009;39(5):742-50.

27. Foxton JM, Stewart ME, Barnard L, Rodgers J, Young AH, O'Brien G, et al. Absence of auditory 'global interference'in autism. Brain. 2003;126(12):2703-9.

28. Pellicano E, Gibson L, Maybery M, Durkin K, Badcock DR. Abnormal global processing along the dorsal visual pathway in autism: a possible mechanism for weak visuospatial coherence? Neuropsychologia. 2005; 43(7):1044-53.

29. Braddick O, Atkinson J, Wattam-Bell J. Normal and anomalous development of visual motion processing: motion coherence and 'dorsal-stream vulnerability'. Neuropsychologia. 2003;41(13):1769-84.

30. Bertone A, Mottron L, Jelenic P, Faubert J. Enhanced and diminished visuospatial information processing in autism depends on stimulus complexity. Brain. 2005;128(10):2430-41.

31. Amunts K, Kedo O, Kindler M, Pieperhoff $P$, Mohlberg H, Shah N, et al. Cytoarchitectonic mapping of the human amygdala, hippocampal region and entorhinal cortex: intersubject variability and probability maps. Anat Embryol (Berl). 2005;210(5-6):343-52.

32. Ball T, Rahm B, Eickhoff SB, Schulze-Bonhage A, Speck O, Mutschler I. Response properties of human amygdala subregions: evidence based on functional MRI combined with probabilistic anatomical maps. PLoS One. 2007;2(3), e307.

33. Roy AK, Shehzad Z, Margulies DS, Kelly AC, Uddin LQ, Gotimer K, et al. Functional connectivity of the human amygdala using resting state fMRI. Neuroimage. 2009;45(2):614.

34. Pitkänen A, Savander V, LeDoux JE. Organization of intra-amygdaloid circuitries in the rat: an emerging framework for understanding functions of the amygdala. Trends Neurosci. 1997;20(11):517-23.

35. Pessoa L. Emotion and cognition and the amygdala: from "what is it?" to "what's to be done?". Neuropsychologia. 2010;48(12):3416-29.

36. Moreno N, González A. Evolution of the amygdaloid complex in vertebrates, with special reference to the anamnio-amniotic transition. J Anat. 2007; 211(2):151-63.

37. Bickart KC, Hollenbeck MC, Barrett LF, Dickerson BC. Intrinsic amygdalacortical functional connectivity predicts social network size in humans. J Neurosci. 2012;32(42):14729-41.

38. Bzdok D, Laird AR, Zilles K, Fox PT, Eickhoff SB. An investigation of the structural, connectional, and functional subspecialization in the human amygdala. Hum Brain Mapp. 2013;34(12):3247-66.

39. LeDoux J. The emotional brain, fear, and the amygdala. Cell Mol Neurobiol. 2003;23(4-5):727-38.

40. Heimer L, Van Hoesen GW. The limbic lobe and its output channels: implications for emotional functions and adaptive behavior. Neurosci Biobehav Rev. 2006;30(2):126-47.

41. Price JL. Comparative aspects of amygdala connectivity. Ann N Y Acad Sci. 2003;985(1):50-8. 
42. Goossens L, Kukolja J, Onur OA, Fink GR, Maier W, Griez E, et al. Selective processing of social stimuli in the superficial amygdala. Hum Brain Mapp. 2009;30(10):3332-8.

43. Pessoa L, Adolphs R. Emotion processing and the amygdala: from a 'low road' to 'many roads' of evaluating biological significance. Nat Rev Neurosci. 2010;11(11):773-83.

44. Adolphs R. The neurobiology of social cognition. Curr Opin Neurobiol. 2001;11(2):231-9.

45. Lord C, Rutter M, Le Couteur A. Autism Diagnostic Interview-Revised: a revised version of a diagnostic interview for caregivers of individuals with possible pervasive developmental disorders. J Autism Dev Disord. 1994; 24(5):659-85.

46. Kort W, Schittekatte $M$, Dekker $P$, Verhaeghe $P$, Compaan E, Bosmans M. Wechsler intelligence scale for children-dutch version. London: Psychological Corporation; 2005.

47. Wechsler D. WAIS-III Nederlandstalige bewerking: Afname-en scoringshandleiding [WAIS-III Dutch version: User manual]. Lisse, The Netherlands: Swets and Zeitlinger; 2000.

48. Oldfield RC. The assessment and analysis of handedness: the Edinburgh inventory. Neuropsychologia. 1971;9(1):97-113.

49. Baron-Cohen S, Wheelwright S, Skinner R, Martin J, Clubley E. The AutismSpectrum Quotient (AQ): evidence from Asperger Syndrome/highfunctioning autism, males and females, scientists and mathematicians. J Autism Dev Disord. 2001;31(1):5-17.

50. Woodbury-Smith MR, Robinson J, Wheelwright S, Baron-Cohen S. Screening adults for Asperger Syndrome using the AQ: a preliminary study of its diagnostic validity in clinical practice. J Autism Dev Disord. 2005;35(3):331-5.

51. Poser BA, Versluis MJ, Hoogduin JM, Norris DG. BOLD contrast sensitivity enhancement and artifact reduction with multiecho EPI: parallel-acquired inhomogeneity-desensitized fMRI. Magn Reson Med. 2006;55(6):1227-35.

52. Chang C, Metzger CD, Glover GH, Duyn JH, Heinze H-J, Walter M. Association between heart rate variability and fluctuations in resting-state functional connectivity. Neuroimage. 2013;68:93-104.

53. Birn RM, Smith MA, Jones TB, Bandettini PA. The respiration response function: the temporal dynamics of fMRI signal fluctuations related to changes in respiration. Neuroimage. 2008:40(2):644-54.

54. Smith SM, Jenkinson M, Woolrich MW, Beckmann CF, Behrens TE, Johansen-Berg $\mathrm{H}$, et al. Advances in functional and structural MR image analysis and implementation as FSL. Neuroimage. 2004;23:S208-S19.

55. Power JD, Barnes KA, Snyder AZ, Schlaggar BL, Petersen SE. Spurious but systematic correlations in functional connectivity MRI networks arise from subject motion. Neuroimage. 2012;59(3):2142-54.

56. Kelly Jr RE, Alexopoulos GS, Wang Z, Gunning FM, Murphy CF, Morimoto SS, et al. Visual inspection of independent components: defining a procedure for artifact removal from fMRI data. J Neurosci Methods. 2010;189(2):233-45.

57. Glover GH, Li TQ, Ress D. Image-based method for retrospective correction of physiological motion effects in fMRI: RETROICOR. Magn Reson Med. 2000;44(1):162-7.

58. O'Reilly JX, Beckmann CF, Tomassini V, Ramnani N, Johansen-Berg H Distinct and overlapping functional zones in the cerebellum defined by resting state functional connectivity. Cereb Cortex. 2010;20(4):953-65.

59. Nichols TE, Holmes AP. Nonparametric permutation tests for functional neuroimaging: a primer with examples. Hum Brain Mapp. 2002;15(1):1-25.

60. Smith SM, Nichols TE. Threshold-free cluster enhancement: addressing problems of smoothing, threshold dependence and localisation in cluster inference. Neuroimage. 2009;44(1):83-98.

61. IBM Corp. IBM SPSS Statistics for Windows, Version 20.0. Armonk: IBM Corp; 2011.

62. Phillips ML, Drevets WC, Rauch SL, Lane R. Neurobiology of emotion perception I: the neural basis of normal emotion perception. Biol Psychiatry. 2003;54(5):504-14.

63. Durston S, Thomas KM, Yang Y, Uluğ AM, Zimmerman RD, Casey B. A neural basis for the development of inhibitory control. Dev Sci. 2002;5(4):F9-F16.

64. Paus T. Mapping brain maturation and cognitive development during adolescence. Trends Cogn Sci. 2005;9(2):60-8.

65. Williams BR, Ponesse JS, Schachar RJ, Logan GD, Tannock R. Development of inhibitory control across the life span. Dev Psychol. 1999;35(1):205.

66. Murphy K, Birn RM, Handwerker DA, Jones TB, Bandettini PA. The impact of global signal regression on resting state correlations: are anti-correlated networks introduced? Neuroimage. 2009;44(3):893-905.
67. Cole DM, Smith SM, Beckmann CF. Advances and pitfalls in the analysis and interpretation of resting-state FMRI data. Front Syst Neurosci. 2010;4:8.

68. Koelsch S, Skouras S, Fritz T, Herrera P, Bonhage C, Küssner MB, et al. The roles of superficial amygdala and auditory cortex in music-evoked fear and joy. Neuroimage. 2013;81:49-60.

69. Sah P, Faber E, De Armentia ML, Power J. The amygdaloid complex: anatomy and physiology. Physiol Rev. 2003;83(3):803-34.

70. Qin S, Young CB, Supekar K, Uddin LQ, Menon V. Immature integration and segregation of emotion-related brain circuitry in young children. Proc Natl Acad Sci U S A. 2012;109(20):7941-6.

71. Schoenbaum G, Chiba AA, Gallagher M. Changes in functional connectivity in orbitofrontal cortex and basolateral amygdala during learning and reversal training. J Neurosci. 2000;20(13):5179-89.

72. Gabard-Durnam LJ, Flannery J, Goff B, Gee DG, Humphreys KL, Telzer E, et al. The development of human amygdala functional connectivity at rest from 4 to 23 years: a cross-sectional study. Neuroimage. 2014;95:193-207.

73. Vissers ME, Cohen MX, Geurts HM. Brain connectivity and high functioning autism: a promising path of research that needs refined models, methodological convergence, and stronger behavioral links. Neurosci Biobehav Rev. 2012;36(1):604-25.

74. Bertone $\mathrm{A}$, Mottron $\mathrm{L}$, Jelenic $\mathrm{P}$, Faubert J. Motion perception in autism: a "complex" issue. J Cogn Neurosci. 2003;15(2):218-25.

75. Bonda E, Petrides M, Ostry D, Evans A. Specific involvement of human parietal systems and the amygdala in the perception of biological motion. J Neurosci. 1996;16(11):3737-44.

76. Gläscher J, Adolphs R. Processing of the arousal of subliminal and supraliminal emotional stimuli by the human amygdala. J Neurosci. 2003;23(32):10274-82

77. McMenamin BW, Marsolek CJ. Can theories of visual representation help to explain asymmetries in amygdala function? Cogn Affect Behav Neurosci. 2013;13(2):211-24

78. Smith SM, Miller KL, Salimi-Khorshidi G, Webster M, Beckmann CF, Nichols TE, et al. Network modelling methods for FMRI. Neuroimage. 2011;54(2):875-91.

\section{Submit your next manuscript to BioMed Central and we will help you at every step:}

- We accept pre-submission inquiries

- Our selector tool helps you to find the most relevant journal

- We provide round the clock customer support

- Convenient online submission

- Thorough peer review

- Inclusion in PubMed and all major indexing services

- Maximum visibility for your research

Submit your manuscript at www.biomedcentral.com/submit
Biomed Central 